\title{
Lifestyle trends in colorectal cancer survivors
}

Several aspects of a healthy lifestyle, including diet, physical activity, and body composition, are associated with reduced mortality in colorectal cancer (CRC) survivors ${ }^{(1)}$; however, limited data on adherence to lifestyle recommendations and changes therein after diagnosis are available. Therefore, the aim of this study is to assess longitudinal changes in adherence to lifestyle recommendations in the first two years following a colorectal cancer diagnosis.

We included 1,057 patients diagnosed with stages I-III CRC between 2010 and 2015 from the prospective COLON cohort study ${ }^{(2)}$. At diagnosis, 6 and 24 months after diagnosis dietary, anthropometric, and physical activity data was collected. A lifestyle score was constructed based on the 2018 World Cancer Research Fund/American Institute of Cancer Research (WCRF/AICR) recommendations for cancer prevention ${ }^{(3)}$. The score range was $0-7$, in which higher scores indicated greater adherence.

Patients had a mean age of 65 (SD 9) years, $63 \%$ were male, $43 \%$ had stage III disease, and $67 \%$ had colon cancer. The mean WCRF/AICR score was 3.7 (SD 0.8) at CRC diagnosis and slightly increased to 3.8 (SD 0.9) 2 years after diagnosis (P<0.0001). At diagnosis, $7 \%$ reported high adherence (score $\geq 5$ ), while this was $11 \%$ at 2 years post-diagnosis (Fig 1). There was a large variation in adherence to the individual recommendations (Fig 1). Overall, small improvements in adherence to some dietary recommendations were seen over time, but adherence to some other recommendations slightly decreased. Overall lifestyle improved with $\geq 0.5$ point in $38 \%$ of CRC survivors in the first two years after diagnosis. However, overall lifestyle deteriorated with $\geq 0.5$ point in $23 \%$ in these two years.
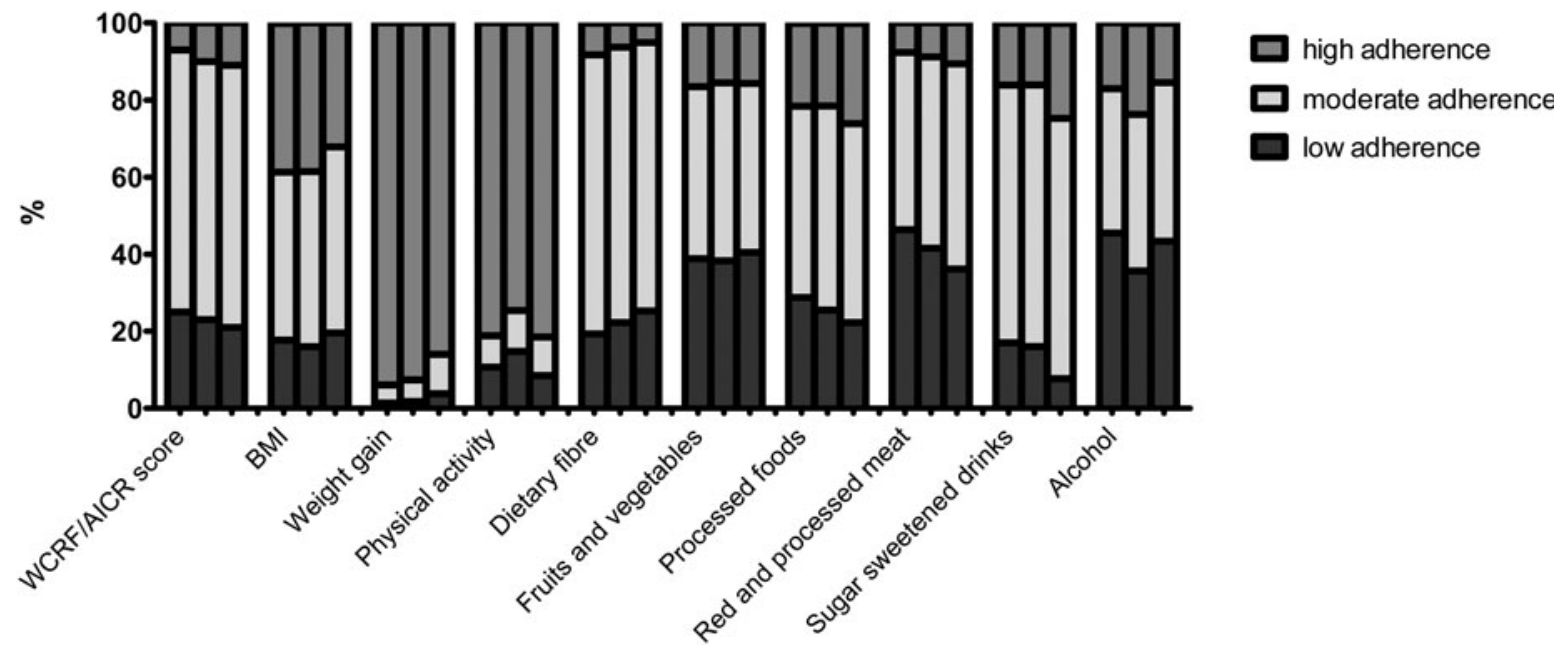

Fig. 1. Adherence to the 2018 World Cancer Research Fund/American Institute for Cancer Research recommendations for cancer prevention at colorectal cancer diagnosis, 6 and 24 months after diagnosis.

In conclusion, preliminary analyses showed that CRC survivors slightly altered their diet and lifestyle after diagnosis. Changes were both positive and negative. Overall, adherence to lifestyle recommendations slightly improved in the first two years after CRC diagnosis. Our next step is to explore which changes in diet and lifestyle are made simultaneously.

1. van Zutphen M, Kampman E, Giovannucci EL et al. (2017) Cur CRC Reports 13, 370-401.

2. Winkels RM, Heine-Broring RC, van Zutphen M et al. (2014) BMC Cancer 14, 374.

3. WCRF / AICR. (2018) Continuous Update Project Expert report 2018. 